\title{
Network Pharmacology-based Elucidation of Molecular Biological Mechanisms of Kanglaite Injection for Treatment of Pancreatic Ductal Adenocarcinoma
}

\section{Bowen Xu}

China Academy of Chinese Medical Sciences Guanganmen Hospital

\section{Wenchao Dan}

China Academy of Chinese Medical Sciences Guanganmen Hospital Jie Lj ( $\sim$ drjieli2007@126.com )

China Academy of Chinese Medical Sciences Guanganmen Hospital https://orcid.org/0000-00023461-8816

\section{Xiaoxiao Zhang}

China Academy of Chinese Medical Sciences Guanganmen Hospital

\section{Luchang Cao}

China Academy of Chinese Medical Sciences Guanganmen Hospital

\section{Shixin Li}

China Academy of Chinese Medical Sciences Guanganmen Hospital

\section{Research}

Keywords: Kanglaite Injection, Pancreatic Ductal Adenocarcinoma, Coix Lacryma-jobi, Network Pharmacology, Mechanism Research

Posted Date: March 30th, 2020

DOI: https://doi.org/10.21203/rs.3.rs-19866/v1

License: (c) (i) This work is licensed under a Creative Commons Attribution 4.0 International License. Read Full License 


\section{Abstract}

Background: Kanglaite injection (KLTi) has shown good clinical efficacy in the treatment of pancreatic ductal adenocarcinoma (PDAC). However, its molecular biological mechanisms are still unclear. This study used network pharmacology approach to investigate the molecular biological mechanisms of KLTi.

Methods: Compounds in KLTi were screened using TCMSP and drug targets were obtained from the DRUGBANK. Next, the GEO database was searched for differentially expressed genes in cancerous tissues and healthy tissues of PDAC patients to identify targets. Subsequently, the protein-protein interaction data of KLTi and PDAC targets were constructed by BisoGenet. A visual analysis was done to extract KLTi candidate genes for PDAC. The candidate genes were enriched using GO and KEGG by Metascape, and the gene-pathway network was constructed to further screen the key genes.

Results: A total of 10 active compounds and 36 drug targets were screened for KLTi, 919 differentially expressed genes associated with PDAC were identified from GEO, and $139 \mathrm{KLTi}$ candidate genes against PDAC were excavated by BisoGenet. The gene-pathway network showed RELA, NFKB1, IKBKG, JUN, $M A P K 1, T P 53$, and $A K T 1$ as the core genes, predicting that $\mathrm{KLTi}$ intervenes in PDAC by acting on these genes.

Conclusions: Our study suggested that KLTi plays an anti-PDAC role by intervening in the cell cycle, inducing apoptosis, regulating protein binding, inhibiting nerve invasion, and down-regulating the NF-KB, MAPK, and PI3K-Akt signaling pathways. In addition, it might also directly participate in the pancreatic cancer pathway. These results provide new evidence and therapeutic direction for subsequent clinical applications and basic research on KLTi in PDAC.

\section{Background}

Pancreatic ductal adenocarcinoma (PDAC) is a malignant tumor of the digestive tract and accounts for about $90 \%$ of all pancreatic cancers (PC). The five-year relative survival rate for PDAC is only $9 \% \square$. The incidence and case fatality rate of PDAC continue to rise, and it is expected to become the second leading cause of cancer-related deaths in $2030^{\square}$. At present, about $90 \%$ of patients with PDAC are diagnosed at an advanced stage, and only $20 \%$ of the patients receive surgical treatment. Moreover, the surgical resection rate of PDAC is low and the five-year survival rate of patients with complete resection is $27 \%$. PDAC is highly metastatic and develops drug resistance easily. Due to low efficacy and high toxicity associated with chemoradiotherapy $\square$, the conventional treatment modalities for PDAC have run into bottleneck. Therefore, it is of great clinical significance and practical value to seek a safe and efficacious alternative or adjuvant therapy for PDAC.

Traditional Chinese Medicine (TCM) has shown to play an active role in the adjuvant treatment of PC by reducing the toxic effects and side effects associated with modern medical treatment ${ }^{\natural}$, improving the 
quality of life, and prolonging the survival of patients. It has also demonstrated sensitization to capecitabine after gemcitabine resistance ${ }^{\square}$.

Kanglaite injection (KLTi) is a neutral oil extracted and isolated from the seeds of the Chinese herbal medicine, Coix lacryma-jobi. It has been widely used in China as an adjuvant therapy for the treatment of various tumors and has shown anti-cachexia and analgesic effects in advanced tumors. KLTi was approved in China in 1995 for the treatment of advanced non-small cell lung cancer and advanced hepatocellular carcinoma ${ }^{\square}$. Apart from its anti-tumor activity, KLTi has also shown to significantly improve immunity. It has been demonstrated that the addition of KLTi to chemoradiotherapy enhanced its efficacy and reduced toxicity. In a phase II clinical trial, the safety and efficacy of KLTi was evaluated in combination with gemcitabine in patients with advanced PC. The results showed that there was a statistically significant difference in progression-free survival in the gemcitabine plus KLTi group compared to the gemcitabine monotherapy group. The median value of the combination group was 114 days, which was higher than that of the gemcitabine group by 57.7 days (hazard ratio [HR]: $0.33 ; 95 \% \mathrm{Cl}$ : $0.14-0.78, P=0.0080)^{\square}$. These results proved the ability of KLTi in improving the efficacy of PDAC treatment. Further exploration of TCM treatment, especially KLTi, is expected to provide benefit in improving the comprehensive treatment of PDAC. KLTi exhibits anti-tumor activity by targeting the G2/M phase of tumor cells, inducing tumor cell apoptosis and necrosis, and inhibiting tumor proliferation and metastasis formation ${ }^{\square}$. However, the specific molecular biological mechanism of KLTi is still not fully understood and needs further investigation.

TCM induces its effects by acting via multiple targets, pathways, and compounds that are involved in various aspects of disease progression. Thus, it is not possible to decode the integrity and multidirectional functionality of TCM treatment by analyzing a single pathway mechanism. The network pharmacology approach emphasizes the multi-way regulation of a specific pathway, analyzes its mutual relationship by constructing the inter-compounds network, and thoroughly examines the key nodes in the network. Network pharmacology has proven to systematically elaborate the material basis and mechanism of action of TCM. Hence, it is currently used to investigate the mechanism of action and new drug development of TCM and its compound prescriptions. Therefore, in this study, the network pharmacology method was used to explore the potential active compounds, key gene targets, basic pharmacological effects, and molecular biological mechanisms of KLTi intervention in PDAC.

\section{Materials And Methods}

\section{Screening of Active Compounds and Targets in KLT}

We identified the total chemical composition of KLTi from the Traditional Chinese Medicine Systems Pharmacology Database and Analysis Platform (TCMSP, Version 2.3 http://Isp.nwu.edu.cn/tcmsp.php).According to the traditional ADME (absorption, distribution, metabolism and excretion) screening principle, there are two core indicators used for screening of compounds, viz. oral bioavailability (OB) and drug likeness (DL). Since KLTi is administered 
intravenously, it did not require screening of $\mathrm{OB}$, that is specific to orally administered drugs. The screening condition for $\mathrm{DL}$ was set as $\geq 0.18$. Compounds related to KLTi that demonstrated anti-tumor activity, as confirmed from previous studies, were collected to supplement and improve the results and obtain candidate compounds. Candidate compounds were then matched to drug targets in the DRUGBANK database (Version 5.1.5, https://www.drugbank.ca/) and corrected to standard genes names, using the Uniprot database (https://www.uniprot.org/). Cytoscape 3.7.2 was employed to construct a compound-target network of KLTi for the selected compounds and targets. In this network, the nodes represented compounds or targets and the edges represented relationship of interactions. The network was analyzed to study the relationship between the important compounds and targets in KLTi, with the help of Cytoscape's built-in network analyzer tool, focusing on the degree of connectivity-the more connected the degree, the greater were the number of involved biological functions, and the higher was its importance. The workflow of the network pharmacology analysis performed in this study is depicted in Fig. 1.

\section{Identification of PDAC-related Targets}

The differential expressed genes in cancerous tissues and healthy tissues of PDAC patients were obtained from the GEO database (https://www.ncbi.nlm.nih.gov/geo/) series (GSE15471, samples: GSM388115-GSM388153 and GSM388076-GSM388114). Disease targets of PDAC were screened under the conditions of adjusted $P$-value $<0.05$ and $|\log F C|>1$, and the gene markers with significant differentially expressed genes corresponded to gene names.

\section{Construction of PPI Network and Screening of Key Targets}

Based on the built-in function of BisoGenet of Cytoscape3.7.2, protein-protein interaction (PPI) network between KLTi and PDAC was constructed and visualized. The intersection network of two PPI networks was extracted by the Merge function of Cytoscape and the attribute values of each node in the intersection network were analyzed using CytoNCA ${ }^{\square}$. The median $\mathrm{k}_{1}$ of the connectivity degree was calculated and all nodes with a connectivity degree greater than 2 times $\mathrm{k}_{1}$ were selected and termed as "Hit hubs." The properties of each node of the Hit hubs network were calculated to obtain three medians $k_{2}, l_{2}$, and $m_{2}$ for connectivity degree centrality (DC), closeness centrality (CC), and betweenness centrality $(B C)$, respectively. All nodes whose node properties were simultaneously greater than $k_{2}, l_{2}$, and $m_{2}$ were screened as candidate genes.

\section{Pathway Enrichment Analysis}

The Metascape platform (http://metascape.org/) integrates several reliable databases such as gene ontology (GO), Kyoto Encyclopedia of Genes and Genomes (KEGG), and Uniprot for pathway enrichment analysis of gene targets and is updated monthly to ensure data accuracy. GO and KEGG analyses of candidate genes can be performed with the help of this platform. GO utilizes three parameters; namely molecular function, biological process, and cellular component to interpret the anti-tumor biological process of candidate genes. KEGG signaling pathway enrichment analysis examines the main anti-tumor 
signaling pathways involved in candidate genes. The top $20 \mathrm{GO}$ and KEGG processes, with significant differences, were screened and the results were visualized and analyzed with $\mathrm{R}$ software. Based on the relevant targets mapped by KEGG results, gene-pathway networks were constructed to further screen key target genes for KLTi treatment of PDAC.

\section{Results}

\section{Active Compounds of KLTi}

A total of 38 compounds were identified in TCMSP, based on the chemical composition of coix lacrymajobi. Candidate compounds with $\mathrm{DL} \geq 0.18$ (18 active compounds) were included in the study. Two compounds, olein and MBOA, that had antitumor activity as reported by previous studies ${ }^{\square}$, were excluded since their $\mathrm{DL}<0.18$. A total of 36 drug targets were matched in the DrugBank database. Of these, 10 active compounds were mapped to the corresponding targets, while 8 compounds did not match to any target (Table 1). A compound-target network was constructed, based on KLTi's active compounds and drug targets (Fig. 2). This network contained 46 nodes (10 compounds in KLTi and 36 drug targets) and 64 edges. The top four key active compounds in KLTi were stigmasterol, mandenol, sitosterol alpha 1 , and isoarborinol and their respective degree and DL values were $26,20,5$, and 5 and $76 \%, 19 \%, 78 \%$, and $77 \%$.

Table 1 The candidate compounds in Kanglaite injection.

\begin{tabular}{|llllll|}
\hline ID & Mol Id & Mol Name & DL & Degree \\
\hline KLT1 & MOL001323 & sitosterol alpha1 & 0.78 & 5 \\
KLT2 & MOL001494 & mandenol & 0.19 & 20 \\
KLT3 & MOL001874 & isoarborinol & 0.77 & 5 \\
KLT4 & MOL001884 & omaine & 0.51 & 1 \\
KLT5 & MOL000298 & ergosterol & 0.72 & 1 \\
KLT6 & MOL000359 & sitosterol & 0.75 & 1 \\
KLT7 & MOL000449 & stigmasterol & 0.76 & 26 \\
KLT8 & MOL000458 & campesterol & 0.72 & 1 \\
KLT9 & MOL008121 & 2-monoolein & 0.29 & 1 \\
KLT10 & MOL000953 & CLR & 0.68 & 3 \\
\hline
\end{tabular}

\section{PDAC-related Targets}

A total of 919 PDAC-related targets were identified from the GEO database. Among these, 709 were upregulated genes and 210 were down-regulated genes. As shown in Fig. 3, a volcano plot was created to 
show the distribution of differentially expressed genes. A heat map of expression for these differential genes is shown in Fig. 4.

\section{Candidate Genes for KLTi Treatment of PDAC}

The PPI networks of PDAC and KLTi were constructed using the BisoGenet function in Cytoscape 3.7.2 software, respectively. We found that the drug targets of KLTi had a relationship with 1,966 targets and 45,343 interrelationships existed among these targets. The PPI network of PDAC putative genes was found to contain 7,983 nodes and 17,6870 edges. The above two PPI networks were merged to reveal the specific targets of KLTi intervention in PDAC. This network, consisting of 1,558 nodes and 40,405 edges, is presented in Fig. 5(A). According to data statistics, the median degree of all nodes was 37, which was filtered with DC $>68$ to obtain Fig. 5(B). The final candidate genes were screened and 139 targets, with DC > 103, BC > 156.4916701, and CC > 0.547414, were identified, as shown in Fig. 5(C).

Since the roles of proteins in PPI networks are reciprocal, they are usually classified as undirected graphs. The presence of regions with high partial density in complex networks of PPI is referred to as community or module. The network inside the module is the potential subnetwork of the PPI network, which has a higher density of subnetwork connections and less regional partial connections. Thus, the module can be considered as a biologically meaningful set, which has two components. First is the protein complex, consisting of multiple proteins to form a complex, which then plays a biological role. The other is the functional module, comprising proteins located in the same pathway but with closer interactions.

Therefore, to analyze the mechanism of KLTi in the treatment of PDAC more precisely, it was necessary to further identify its intrinsic module after obtaining the core PPI network. The module was obtained by analyzing the interaction relationship through the molecular complex detection algorithm, as shown in Fig. 6. Based on the $p$ value, the biological processes of the three best scores in the PPI network and module were retained and functionally described. The functional descriptions are shown in Table 2 .

Table 2 Description of potential module functions within protein-protein interaction network for Kanglaite injection treatment pancreatic ductal adenocarcinoma (top 3). 


\begin{tabular}{|llllc|}
\hline Color & MCODE & GO & Description & Log10P \\
\hline Red & MCODE_1 & GO:0003735 & structural constituent of ribosome & -29.7 \\
\hline Red & MCODE_1 & GO:0005198 & structural molecule activity & -21.6 \\
\hline Red & MCODE_1 & GO:0019843 & rRNA binding & -11.2 \\
\hline Blue & MCODE_2 & GO:0019904 & protein domain specific binding & -11.5 \\
\hline Blue & MCODE_2 & GO:0043021 & ribonucleoprotein complex binding & -9.3 \\
\hline Blue & MCODE_2 & GO:0031625 & ubiquitin protein ligase binding & -8.4 \\
\hline Green & MCODE_3 & GO:0031625 & ubiquitin protein ligase binding & -18.4 \\
\hline Green & MCODE_3 & GO:0044389 & ubiquitin-like protein ligase binding & -18.1 \\
\hline Green & MCODE_3 & GO:0019904 & protein domain specific binding & -11.7 \\
\hline Purple & MCODE_4 & GO:0008134 & transcription factor binding & -13.8 \\
\hline Purple & MCODE_4 & GO:0003682 & chromatin binding & -11.6 \\
\hline Purple & MCODE_4 & GO:0001085 & RNA polymerase Il transcription factor binding & -9.8 \\
\hline Orange & MCODE_5 & GO:0003712 & transcription coregulator activity & -6.1 \\
\hline Orange & MCODE_5 & GO:0031625 & ubiquitin protein ligase binding & -5.6 \\
\hline Orange & MCODE_5 & GO:0044389 & ubiquitin-like protein ligase binding & -5.5 \\
\hline
\end{tabular}

\section{GO and KEGG Pathway Enrichment}

Metascape platform was used to perform GO and KEGG pathway analysis of the 139 identified candidate genes. The $\mathrm{GO}$ results of candidate genes showed that a total of 1,762 $\mathrm{GO}$ terms were significantly enriched, including 1,520 in biological processes, 133 in cellular compositions, and 109 in molecular functions.

According to the $\log 10$ (false discovery ratio [FDR]) value ranking, the top 20 genes were selected (Fig. 7). The top biological processes were apoptotic signaling pathway, regulation of cell cycle process, regulation of cellular response to stress, and regulation of protein modification. The top cellular compounds were nuclear chromosome, focal adhesion, cell-substrate adherens junction, cell-substrate junction, cytosolic ribosome, and nuclear chromosome part. The top molecular functions were ubiquitin protein ligase binding, protein domain specific binding, transcription factor binding, kinase binding, histone deacetylase binding, and structural constituent of ribosome enrichment.

KEGG results for candidate genes revealed 119 significantly enriched pathways. As shown in Fig. 8, the top 20 genes were selected to be represented in the bubble diagram. The scatter diagram includes 
signaling pathways such as viral carcinogenesis, pathways in cancer, cell cycle, MAPK signaling pathway, PI3K-Akt signaling pathway, neurotrophin signaling pathway, pancreatic cancer, and apoptosis.

\subsection{Gene-Pathway Network}

The gene-pathway network was constructed based on the significant difference in KEGG pathways and genes that regulated these pathways. It included 20 signaling pathways, 57 genes, and 253 relationships (Fig. 9). From the network, it was observed that RELA and NFKB1 had the largest degree (19). The other genes with large degrees were IKBKG, JUN, MAPK1, AKT1, and TP53 $(17,16,15,12$, and 10, respectively). These genes might be the core target genes for KLTi intervention in PDAC.

\section{Discussion}

TCM has been used to treat various diseases for thousands of years. Numerous studies have indicated that the combination of TCM and modern medicine can play an indispensable role in improving efficacy and reducing toxicity associated with cancer treatment ${ }^{\square}$. KLTi is a medicinal herb extracted from coix lacryma-jobi, which acts by supplementing Qi and nourishing Yin and removing stasis. TCM focuses on 'syndrome differentiation and treatment.' KLTi has demonstrated clinical effect in patients with PDAC with splenasthenic hygrosis and deficiency of both Qi and Yin. In previous studies, KLTi monotherapy has shown antitumor activity in mouse models of PDAC by inhibiting tumor cell proliferation and inducing apoptosis $\square$. In addition, a meta-analysis showed that the combination of KLTi and radiochemotherapy is more effective than radiochemotherapy alone in the treatment of advanced PDAC. The combination treatment not only improved the 1-year overall survival rate, overall response, and disease control rate, but also enhanced the quality of life and relieved pain. In addition, it alleviated the adverse events caused by chemoradiotherapy, such as gastrointestinal side effects, nephrotoxicity, leukopenia, thrombocytopenia, and myelosuppression?.

TCM injection is a preparation composed of multiple compounds corresponding to many targets. Further, there may be synergistic or antagonistic effects between targets. Hence, it is difficult to elucidate the mechanism of action of KLTi using conventional approaches that use drug-target-disease framework. Network pharmacology is based on various types of biological information databases. Through the network analysis of drugs and diseases, it is possible to explain the overall mechanism of action and compound information of TCM and related compounds. Network pharmacology emphasizes the study of multi-target pathways, which is consistent with the overall concept of TCM.

\section{Potential Active Compounds}

The top four compounds obtained from the compound-target network of KLTi were stigmasterol, mandenol, sitosterol alpha1, and isoarborinol. The degree values of stigmasterol and mandenol were found to be significantly superior compared to others. Moreover, these compounds exhibited a high degree of biological activity and could map multiple drug targets. Hence, they were designated as the core compounds in KLTi. Stigmasterol and sitosterol alpha1 belong to the class of phytosterols, which 
are chemicals with tumor prevention and anticancer effects, and are present in a variety of medicinal plants. Phytosterols can also induce significant reduction in cell viability and induce apoptotic death of tumor cells ${ }^{\square}$.

Stigmasterol has demonstrated anti-inflammatory, anticancer, anti-allergic, and immune regulation effects. Stigmasterol inhibited lipopolysaccharide (LPS)-induced innate immune responses in murine models ${ }^{\square}$, significantly reduced the transcript level of TNF-a, destroyed tumor angiogenesis, and reduced the chance of metastasis $\llbracket$. Studies have reported that stigmasterol induces mitochondrial-mediated apoptosis to inhibit tumor cell proliferation by interfering with Bax and Bcl-2 expression. It also inhibits tumor cell metastasis and induces $\mathrm{G} 2 / \mathrm{M}$ cell cycle arrest in a dose-dependent manner ${ }^{\square}$.

Although there is insufficient data on sitosterol alpha1, related subclasses such as $\beta$-sitosterol and $\gamma^{-}$ sitosterol have demonstrated obvious anticancer effects. Several in vitro and in vivo studies of $\beta$ sitosterol combined with gemcitabine have revealed that $\beta$-sitosterol could increase the expression of Bax protein and reduce the expression of $\mathrm{Bcl}-2$ protein, induce $\mathrm{G} 0 / \mathrm{G} 1$ phase arrest and apoptosis, and suppress NF-KB activity to effectively inhibit PC cell growth. It also inhibits the invasion and metastasis of anti-tumor cells, and the combination with gemcitabine showed a significant synergistic effect ${ }^{\square}$. In addition, $y$-sitosterol exerts anticancer activity by inhibiting the growth of tumor cells by blocking them in the $\mathrm{G} 2 / \mathrm{M}$ phase and inducing apoptosis ${ }^{\square}$.

Modern pharmacological studies have confirmed that isoarborinol can be used to improve anxiety, depression, and pain. Moreover, it has an auxiliary effect on the clinical symptoms which are prone to occur during the development of cancer treatment ${ }^{\natural}$. In case of mandenol, there is currently no relevant clinical or experimental research data available.

\section{Potential Genetic Targets}

From the "gene-pathway" network, it was found that RELA, NFKB1, IKBKG, JUN, MAPK1, TP53, and AKT1 were the genes with the highest interactions and were identified as potential gene targets for KLTi intervention in PDAC. Among these genes, RELA, NFKB1, and IKBKG are all components of the NF-kappa B signaling pathway. The NF-kappa B signaling pathway is one of the major signaling pathways linking cancer to inflammation. This classical pathway is activated when the cells are exposed to inflammatory cytokines, such as TNFa and IL-1 or in response to inflammatory signals, such as LPS ${ }^{\square}$. In addition to inhibiting tumor cell proliferation and metastasis, NF-KB also interferes with inflammation $\square$. Specifically, for PDAC with pancreatitis, intervention with NF-kappa B signaling pathway can simultaneously result in tumor and tumor-related inflammation. NF-KB also helps TNF-a to induce epithelial-mesenchymal transition (EMT) and complete angiogenesis and metastasis. Thus, KLTi could curb tumor progression by inhibiting NF-kappa B signaling pathway.

RELA can promote PDAC progression by activating proliferation or migration-related gene expression. This suggests that the overexpression of RELA promotes proliferation and metastasis of PDAC cells. Binding of miR-302a-3p to RELA, inhibited RELA expression as well as PDAC cell proliferation and 
migration $^{\square}$. Tumor suppression, mediated by oncogene-induced senescence (OIS), is thought to play a protective role in the development of PDAC. In the Kras-driven PDAC mouse model, Lesina et al demonstrated that RELA reinforced OIS to inhibit carcinogenesis ${ }^{\square}$. However, genetically disabling OIS can cause RELA to promote tumor proliferation; thus revealing a dual role of RELA in PDAC carcinogenesis.

NFKB1 is part of the NF-kappa B signaling pathway. Low expression of MUC4 inhibited the expression of NFKB1, thereby down-regulating the NF-kappa B signaling pathway to inhibit the migration and invasion of PDAC cells and impaired the migration of PDAC cells along the nerve ${ }^{\square}$. As a transcription factor, NFKB1 is responsible for regulating immune response, inflammatory processes and cell growth, and is closely related to the risk of tumor occurrence and disease prognosis ${ }^{\square}$. NFKB1 also affects susceptibility to tumors, and its polymorphisms significantly increase susceptibility to cancer in Asians ${ }^{\square}$. Several researchers have pointed out the important role of NFKB1 as an inhibitor of inflammation, cancer, and aging ${ }^{\square}$.

IKBKG binds and regulates IKB kinase (IKK), which is required for NF-kappa B signaling pathway activation. The IKBKG -binding domain peptide (NBDP) of IKK has been found to inhibit NF-KB activation and increase the cleavage of PARP and Caspase 3 in the apoptotic pathway to promote apoptosis in PDAC cells. It has also been reported that NBDP promotes sensitivity of PDAC to gemcitabine. Therefore, regulating the activity of IKBKG can help in controlling PDAC, and also be used to increase the sensitivity to chemotherapy ${ }^{\square}$.

Studies have shown that JUN may be involved in the growth of PDAC cells ${ }^{\square}$. Inhibition of JUN also inhibits histone deacetylase inhibitors (HDACi)-induced inflammatory gene expression and tumorsupported response in fibroblasts. This indicates that the combination of HDACi and chemical inhibitors of the JUN attenuates the inflammatory phenotype of fibroblasts, and may improve the anti-tumor efficacy of HDACi in PDAC, and even in other solid tumors ${ }^{\square}$. In addition, it plays an important role in regulating the K-Ras pathway by interfering with JUN and exerting anti-inflammatory activity and inhibiting PDAC metastasis $\square$.

MAPK1 is an important protein kinase and regulatory factor in the MAPK signaling pathway which can enhance the invasion and proliferation of PDAC cells ${ }^{\square}$, and also reverse the inhibitory effect of miR-212 on tumor cell proliferation and apoptosis $\llbracket$. MAPK1 can also induce PDAC EMT, which greatly promotes the migration and invasion of cancer cells ${ }^{\square}$. Therefore, inhibition of MAPK 1 expression is also a potential target for future oncological research.

AKT1, a serine/threonine protein kinase, has been identified as an oncogene in a variety of cancers, including PDAC. Studies have confirmed that activated AKT1 accelerates the occurrence and development of PDAC. It mediates proliferation inhibition and induces apoptosis of PDAC cells by inhibiting AKT1 expression ${ }^{\square}$. Phosphorylation of AKT1 also increases the risk of cachexia in the PDAC population, which is strongly associated with disease prognosis ${ }^{\square}$. 
TP53 mutations occur in more than 75\% of PC patients, and mutated TP53 promotes EMT and tumor cell invasion ${ }^{\square}$. TP53 is a driver gene that is essential for the proliferation and metastasis of PDAC, and the expression of TP53 results in shorter disease free survival (HR: 1.33; 95\% Cl, 1.02-1.75; $P=0.04)^{\square}$. In addition, some studies have found that the mutation of TP53 is closely related to the occurrence of malignant intraductal papillary mucinous neoplasms (IPMNs) ${ }^{\square}$. Therefore, silencing or inactivation of TP53 will prevent the further development of IPMN to PDAC and improve the prognosis of patients.

\section{Gene Ontology}

The GO molecular function of the core genes showed that these genes are mainly enriched in enzyme binding, transcription factor binding, and receptor binding. There are many transcription factors in the gene targets of KLTi for the treatment of PDAC, as well as in the binding processes of enzyme, transcription factor, and receptor protein. These factors play an important role by intervening the binding of these proteins. For example, myc-associated zinc-finger protein (MAZ) is the transcription factor involved in the transcription initiation and termination. Deregulation of MAZ expression is associated with the progression of PDAC and increases CRAF-ERK signal. It is mediated through p21-activated protein kinase (PAK) and protein kinase $B(A K T / P K B)$ signaling cascades to enhance the invasion of PDAC cells $\square$. There are also some transcription factors that promote the malignant transformation of PDAC ${ }^{\square}$. Thus, intervention of the binding of transcription factors might have some interference effects on PDAC.

The GO biological process of core genes was mainly enriched in apoptotic signaling pathway, cell cycle, and metabolic process. As far as the function of potential gene targets in this study is concerned, KLTi inhibited the proliferation and induced apoptosis in human PC xenografts through various mechanisms, such as cell cycle arrest in G2/M phase, down-regulating the expression of phosphorylated Akt and mTOR, and regulating the PI3K/Akt/mTOR signaling pathway ${ }^{\text {. }}$

The GO cellular components of core genes were mainly enriched in nuclear chromosomes, perinuclear regions, and ribosomes. Previous studies have shown that KLTi reduces NF-KB levels in the nucleus. Additionally, it reduces the expression of $\mathrm{IKBa}$, IKK, and EGFR in tumor cells and the overall cytoplasm. This corresponds to the role of KLTi at the nuclear and cytoplasmic levels ${ }^{\square}$. Enzyme receptors and transcription factors rely on protein to function, and proteins are, in turn, synthesized by ribosomes. Thus, intervening the functioning of the ribosomes that synthesize the corresponding protein may have some benefits in controlling the disease. For example, connexins are a group of tumor suppressor genes, amongst which Connexin 43 (Cx43) is the most widely expressed gene. The up-regulation of $\mathrm{Cx} 43$ expression can improve the sensitivity of many tumors to chemotherapy and radiotherapy, and this site of action may be present in cytoplasmic ribosomes ${ }^{\square}$.

\section{KEGG pathway}

Results from KEGG analysis showed that the regulatory pathways of KLTi for PDAC are mainly enriched in viral carcinogenesis, cancer pathways, cell cycle, MAPK signaling pathway, PI3K-Akt signaling pathway, 
and neurotrophin signaling pathway. It is worth noting that KLTi can act directly on PC, in addition to intervening in signaling pathways that affect tumor development. This observation is sufficient to confirm that KLTI has an adequate scientific basis in the treatment of PDAC.

It has been proven that many malignant tumors are associated with virus infection. Virus produce prion protein that induce reprogramming and genomic instability, including the accumulation of mutations, aberrations, and DNA damage ${ }^{\square}$. Most DNA oncogenic viruses are often involved in cell proliferation and regulation of the cell cycle by binding to $\mathrm{p} 53$ and pRB tumor suppressor proteins, thereby inducing tumor formation $\llbracket$. Studies have shown a correlation between HBV infection and the occurrence of PC,and poor prognosis. The $X$ protein released by HBV significantly enhances cell proliferation and migration, induces EMT, up-regulates PI3K-Akt and MAPK signaling pathways, render PDAC malignant, and promotes disease progression".

Regulating cell cycle also plays a role in the development of PDAC. A variety of genes and proteins are involved in this process. Usami et al ${ }^{\mathbb{1}}$ observed that the class ${ }^{2}$ HDACi could inhibit the activation of FOXO3, thus inhibiting the growth of PC cells. The combination of class $\mathbb{~}$ a HDACi with the proteasome inhibitor, carfilzomib, could have a synergistic effect on the FOXO3 activation, thus resulting in G1/S arrest in AsPC-1 cells.

Peripancreatic nerve invasion is an important oncological feature of PDAC and is closely related to disease prognosis. KLTi can also act on the neurotrophin signaling pathway to intervene nerve invasion. Perineural invasion is associated with a variety of neurotrophic factors produced by neural tissue inside and outside the pancreas which bind to specific receptors resulting in autophosphorylation and activation of multiple signaling pathways, such as MAPK, PI3K-Akt, and NF-kappa $B^{\square}$. The nerve growth factor promotes the spread of PC cells by autocrine and/or paracrine mechanisms through MAPK-mediated phosphorylation ${ }^{\square}$. It also activates the ERK/CD133 signaling cascade, resulting in enhanced tumor cell invasion, and plays a key role in perineural invasion of $\mathrm{PC}^{\square}$. Studies have confirmed that regulating NFkappa B signaling pathway through activating IKK plays an important role in mediating EMT and inducing neural invasion".

The activation of the MAPK signaling pathway is crucial for PDAC proliferation and metastasis. This pathway is involved in the regulation of various biological activities through three major proteins, ERK1/2, p38MAPK, and MKK4 $4^{\square}$. Yan et al ${ }^{\square}$. compared the expression of $p$-ERK1/2 between pancreatic cancerous tissues and normal cells. They found that the expression of $p$-ERK1/2 in pancreatic tissues was significantly increased. In vivo and in vitro experiments confirmed that the inhibition of ERK $1 / 2$ expression reduced EMT, activated cancer-related autophagy, and decreased cell proliferation and migration in human PC cells. In another study ${ }^{\square}$, it was shown that the p38MAPK inhibitor, VCP979, could regulate the MAPK/NF-KB signaling pathway, reduce inflammation, and inhibit EMT to exert antitumor effect. Further, MKK4 was associated with high proliferation of tumor cells and promoted rapid proliferation of PDAC cells. 
The PI3K-Akt signaling pathway is a key pathway that promotes tumor cell proliferation, invasion, metastasis, and drug resistance. As one of the substrates of Akt, Girdin enhanced phosphorylation of Akt and induced activation. Wang et al ${ }^{[}$. found that Girdin showed high expression in PDAC and was involved in the regulation of tumor cell metastasis, angiogenesis, and autophagy. Silencing of the Girdin gene resulted in decreased levels of p-Akt and p-PI3K, and inhibition of the PI3K-Akt signaling pathway, thereby increasing apoptosis and inducing cell cycle arrest in tumor cells. PI3K-Akt signaling pathway is closely related to the abnormal express of IncRNA. Studies ${ }^{\square}$ have shown that the expression of IncRNA small nucleolar RNA host gene1 elevates the expression of PI3K and phosphorylated Akt, which in turn activates the PI3K-Akt signaling pathway to promote cell proliferation, inhibits apoptosis, and enhances invasion in PDAC. The expression of IncRNA AB209630 inhibited the PI3K-Akt signaling pathway in gemcitabine-resistant PDAC cells and reduced the proliferation of resistant cells to improve the sensitivity to chemotherapy ${ }^{\square}$.

It is worth noting that both MAPK and PI3K-Akt are important signaling pathways for the transduction of membrane receptor signals into cells, and there exists an interaction of receptor signals in the two pathways ${ }^{\square}$. Ras, an upstream molecule of MAPK, can induce the activation of Akt, and p38MAPK can act between PI3K and Akt and thus play an important role in Akt phosphorylation. Similarly, the activity of PI3K has an important induction effect on the activity of the Ras/MAPK pathway, and Akt can also negatively regulate the Ras/MAPK pathway by phosphorylating Raf $\square$.

PI3K-Akt and NF-KB signaling pathways also have an interaction effect. Akt activates Ikk, by phosphorylation, then releases NF-KB from the cytoplasm for nuclear translocation, activates downstream gene expression, and participates in the regulation of NF-KB pathway $\square$.

\section{Conclusions}

In this study, we used the network pharmacology approach to conduct a preliminary investigation on the mechanism of action of KLTi in PDAC. Results indicated that KLTi achieved a synergistic effect through multiple compounds, targets, and pathways. The four effective compounds of KLTi, namely stigmasterol, sitosterol alpha1, and isoarborinol, were involved in regulating most of the PDAC targets. Among the potential targets of KLTi intervention in PDAC, RELA, NFKB1, and IKBKG showed good NF-kappa B signaling activity and could regulate this pathway to exert anti-PDAC activity. The other genes (JUN, MAPK1, TP53, and AKT1) mostly exerted anti-tumor activity by regulating the transcription factors and inhibiting protease activity. Results from the GO and KEGG analysis showed that KLTi exerted anti-PDAC effects by regulating cell cycle, inducing apoptosis, and participating in cancer-related pathways, neurotrophin signaling pathway, MAPK signaling pathway, and PI3K-Akt signaling pathway and their interactions. Through the mining of network pharmacology, this study found that KLTi treatment of PDAC could directly regulate the pancreatic cancer pathway, thus providing scientific evidence for the rational application of KLTi for PDAC in clinical practice. These results also provide a theoretical foundation for subsequent basic experiments. 


\section{Availability Of Data And Materials}

The data and materials used to support the findings of this study are available from the corresponding author upon request.

\section{Abbreviations}

KLTi: Kanglaite Injection

PDAC: Pancreatic Ductal Adenocarcinoma

TCMSP: Traditional Chinese Medicine Systems Pharmacology Database and Analysis Platform

PC: Pancreatic Cancers

TCM: Traditional Chinese Medicine

HR: Hazard Ratio

ADME: Absorption, Distribution, Metabolism and Excretion

OB: Oral Bioavailability

DL: Drug Likeness

PPI: Protein-Protein Interaction

DC: Degree Centrality

CC: Closeness Centrality

BC: Betweenness Centrality

GO: Gene Ontology

KEGG: Kyoto Encyclopedia of Genes and Genomes

FDR: False Discovery Ratio

LPS: Lipopolysaccharide

EMT: Epithelial-mesenchymal transition

OIS: Oncogene-induced senescence

IKK: IKB kinase 
NBDP: IKBKG -binding domain peptide

HDACi: Histone deacetylase inhibitors

MAZ: Myc-associated zinc-finger protein

Cx43: Connexin 43

IPMNs: intraductal papillary mucinous neoplasms

PAK: p21-activated protein kinase

PKB: protein kinase $B$

RELA: RELA proto-oncogene, NF-kB subunit

NFKB1: Nuclear factor kappa B subunit 1

IKBKG: Inhibitor of nuclear factor kappa B kinase regulatory subunit gamma

JUN: Jun proto-oncogene, AP-1 transcription factor subunit

MAPK1: Mitogen-activated protein kinase 1

TP53: Tumor protein p53

AKT1: AKT serine/threonine kinase 1

EGFR: Epidermal growth factor receptor

\section{Declarations}

\section{Availability of data and materials}

The data and materials used to support the findings of this study are available from the corresponding author upon request.

\section{Acknowledgments}

We would like to thank Editage for English language editing.

\section{Funding}

This research was supported by the 2018 National Key Research and Development Program "Modernization Research of Traditional Chinese Medicine"(2018YFC1707405) and National Natural Science Foundation of China $(81273946,81473463,81774289)$. 


\section{Affiliations}

${ }^{1}$ Guang'anmen Hospital, China Academy of Chinese Medical Sciences, Beijing 100053, China

Bowen Xu, Wenchao Dan, Jie Li, Xiaoxaio Zhang, Luchang Cao \& Shixin Li

${ }^{2}$ Graduate School of Beijing University of Traditional Chinese Medicine, Beijing 100029, China

Bowen Xu, Wenchao Dan \& Shixin Li

\section{Contributions}

XBW and DWC came up with idea and designed study with LJ. XBW and DWC perform the main analysis and draft the manuscript. ZXX assisted discussion and analysis. CLC and LSX assisted in preparing the manuscript. All authors wrote, read, and approved the final manuscript.

\section{Authors information}

Bowen $\mathrm{Xu}$ and Wenchao Dan are equal first authors.

\section{Corresponding authors}

Correspondence should be addressed to: Jie Li, drjieli2007@126.com.

\section{Ethics declarations}

\section{Ethics approval and consent to participate}

This article does not contain any animal experiments.

\section{Consent for publication}

The manuscript is approved by all authors for publication.

\section{Competing interests}

The authors declare that there are no conflicts of interest regarding the publication of this paper.

\section{Publisher's Note}

Springer Nature remains neutral with regard to jurisdictional claims in published maps and institutional affiliations.

\section{References}


1. Siegel R L, Miller K D, Jemal A. Cancer statistics, 2019[J]. CA: A Cancer Journal for Clinicians, 2019, 69(1): 7- 34.

2. Rahib L, Smith B D, Aizenberg R, et al. Projecting Cancer Incidence and Deaths to 2030: The Unexpected Burden of Thyroid, Liver, and Pancreas Cancers in the United States[J]. Cancer Research, 2014, 74(14): 2913- 2921.

3. McGuigan A, Kelly P, Turkington R C, et al. Pancreatic cancer: A review of clinical diagnosis, epidemiology, treatment and outcomes[J]. World Journal of Gastroenterology, 2018, 24(43): 48464861.

4. Zhan $\mathrm{HX}, \mathrm{Xu} \mathrm{J} \mathrm{W}, \mathrm{Wu}$ D, et al. Neoadjuvant therapy in pancreatic cancer: a systematic review and meta-analysis of prospective studies[J]. Cancer Medicine, 2017, 6(6): 1201- 1219.

5. Yang X, Hao J, Zhu C H, et al. Survival Benefits of Western and Traditional Chinese Medicine Treatment for Patients With Pancreatic Cancer[J]. Medicine, 2015, 94(26): 1008.

6. Saif M W, Li J, Lamb L, et al. First-in-human phase II trial of the botanical formulation PHY906 with capecitabine as second-line therapy in patients with advanced pancreatic cancer[J]. Cancer Chemotherapy \& Pharmacology, 2014, 73(2): 373- 380.

7. Qi F, Zhao L, Zhou A, et al. The advantages of using traditional Chinese medicine as an adjunctive therapy in the whole course of cancer treatment instead of only terminal stage of cancer[J]. BioScience Trends, 2015, 9(1): 16- 34.

8. Schwartzberg L S, Arena F P, Bienvenu B J, et al. A Randomized, Open-Label, Safety and Exploratory Efficacy Study of Kanglaite Injection (KLTi) plus Gemcitabine versus Gemcitabine in Patients with Advanced Pancreatic Cancer[J]. Journal of Cancer, 2017, 8(10): 1872- 1883.

9. Lu Y, Li C S, Dong Q. Chinese herb related molecules of cancer-cell-apoptosis: a minireview of progress between Kanglaite injection and related genes[J]. Journal of Experimental \& Clinical Cancer Research, 2008, 27(1): 31.

10. Tang Y, Li M, Wang J, et al. CytoNCA: A cytoscape plugin for centrality analysis and evaluation of protein interaction networks[J]. Biosystems, 2015, 127: 67- 72.

11. Wang B L. Network Pharmacology Study of Anticancer Mechanism of Three Main Components in Kanglaite Injection[J]. Chinese Journal of Modern Applied Pharmacy, 2019, 36(1): 58- 63.

12. Wang Z X, Qi F H, Cui Y G, et al. An update on Chinese herbal medicines as adjuvant treatment of anticancer therapeutics[J]. BioScience Trends, 2018, 12(3): 220- 239.

13. Liu $Y$, Zhang W, Wang $X \mathrm{~J}$, et al. Antitumor effect of Kanglaite ${ }^{\circledR}$ injection in human pancreatic cancer xenografts[J]. BMC Complementary \& Alternative Medicine, 2014, 14: 228.

14. Liu J, Yu L, Ding W. Efficacy and safety of Kanglaite injection combined with radiochemotherapy in the treatment of advanced pancreatic cancer: A PRISMA-compliant meta-analysis[J]. Medicine, 2019, 98(32): 16656.

15. Alvarez-Sala A, Attanzio A, Tesoriere $L$, et al. Apoptotic effect of a phytosterol-ingredient and its main phytosterol ( $\beta$-sitosterol) in human cancer cell lines[J]. International journal of food sciences and nutrition, 2019, 70(3): 323-334. 
16. Antwi A O, Obiri D D, Osafo N, et al. Stigmasterol inhibits lipopolysaccharide-induced innate immune responses in murine models[J]. International Immunopharmacology, 2017, 53: 105- 113.

17. Kangsamaksin $T$, Chaithongyot $S$, Wootthichichairangsan $C$, et al. Lupeol and stigmasterol suppress tumor angiogenesis and inhibit cholangiocarcinoma growth in mice via downregulation of tumor necrosis factor-a[J]. PLOS ONE, 2017, 12(12): 189628.

18. Li K, Yuan D, Yan R, et al. Stigmasterol exhibits potent antitumor effects in human gastric cancer cells mediated via inhibition of cell migration, cell cycle arrest, mitochondrial mediated apoptosis and inhibition of JAK/STAT signalling pathway[J]. JOURNAL OF BUON, 2018, 23(5): 1420- 1425.

19. Cao Z Q, Wang X X, Lu L, et al. $\beta$-Sitosterol and Gemcitabine Exhibit Synergistic Anti-pancreatic Cancer Activity by Modulating Apoptosis and Inhibiting Epithelial-Mesenchymal Transition by Deactivating Akt/GSK-3ß Signaling[J]. Frontiers in Pharmacology, 2019, 9: 1525.

20. Sundarraj S, Thangam R, Sreevani V, et al. $Y$-Sitosterol from Acacia nilotica L. induces G2/M cell cycle arrest and apoptosis through c-Myc suppression in MCF-7 and A549 cells[J]. Journal of Ethnopharmacology, 2012, 141(3): 803- 809.

21. Luz, D A, Pinheiro A M, Silva M L, et al. Ethnobotany, phytochemistry and neuropharmacological effects of Petiveria alliacea L. (Phytolaccaceae): A review[J]. Journal of Ethnopharmacology, 2016, 5: 182- 201.

22. Kabacaoglu D, Ruess D A, Ai J, et al. NF-kB/Rel Transcription Factors in Pancreatic Cancer: Focusing on RelA, c-Rel, and RelB[J]. Cancers, 2019, 11(7): 937.

23. Ben-Neriah Y, Karin M. Inflammation meets cancer, with NF-KB as the matchmaker[J]. Nature Immunology, 2011, 12(8): 715- 723.

24. Luo Z, Yi Z J, Ou Z L, et al. RELA/NEAT1/miR-302a-3p/RELA feedback loop modulates pancreatic ductal adenocarcinoma cell proliferation and migration[J]. Journal of cellular physiology, 2018, 234(4): 3583- 3597.

25. Lesina $M$, Wörmann S M, Morton J, et al. RelA regulates CXCL1/CXCR2-dependent oncogene-induced senescence in murine Kras-driven pancreatic carcinogenesis[J]. Journal of Clinical Investigation, 2016, 126(8): 2919- 2932.

26. Wang L, Zhi X, Zhu Y, et al. MUC4-promoted neural invasion is mediated by the axon guidance factor netrin-1 in PDAC[J]. Oncotarget, 2015, 6(32): 33805- 33822.

27. Pectasides D, Kotoula V, Papaxoinis G, et al. Expression Patterns of Growth and Survival Genes with Prognostic Implications in Advanced Pancreatic Cancer[J]. Anticancer Research, 2016, 36(12): 63476356.

28. Luo Y Q, Wang D, Gong T, et al. An updated meta-analysis of 37 case-control studies on the association between NFKB1 -94ins/del ATTG promoter polymorphism and cancer susceptibility[J]. Oncotarget, 2016, 7(36): 58659- 58670.

29. Cartwright T, Perkins N D, Wilson C. NFKB1: A Suppressor of Inflammation, Ageing and Cancer[J]. FEBS Journal, 2016, 283(10): 1812- 1822. 
30. Zhuang Z, Li H, Lee H, et al. NEMO peptide inhibits the growth of pancreatic ductal adenocarcinoma by blocking NF-KB activation[J]. Cancer Letters, 2017, 411: 44- 56.

31. Meggiato T, Calabrese F, De Cesare C M, et al. C-JUN and CPP32 (CASPASE 3) in Human Pancreatic Cancer: Relation to Cell Proliferation and Death[J]. Pancreas, 2003, 26(1): 65- 70.

32. Nguyen A H, Elliott I A, Wu N, et al. Histone deacetylase inhibitors provoke a tumor supportive phenotype in pancreatic cancer associated fibroblasts[J]. Oncotarget, 2016, 8(12): 19074- 19088.

33. Tjomsland V, Bojmar L, Sandström P, et al. IL-1 a Expression in Pancreatic Ductal Adenocarcinoma Affects the Tumor Cell Migration and Is Regulated by the p38 MAPK Signaling Pathway[J]. PloS One, 2013, 8(8): 70874.

34. Karakhanova S, Golovastova M, Philippov P P, et al. Interlude of cGMP and cGMP/Protein Kinase G Type 1 in Pancreatic Adenocarcinoma Cells[J]. Pancreas, 2014, 43(5): 784- 794.

35. Hu B, Jin X B, Wang J B. MicroRNA-212 Targets Mitogen-Activated Protein Kinase 1 to Inhibit Proliferation and Invasion of Prostate Cancer Cells[J]. Oncology Research Featuring Preclinical and Clinical Cancer Therapeutics, 2018, 26(7): 1093- 1102.

36. Hu J, Li L, Chen H, et al. MiR-361-3p regulates ERK1/2-induced EMT via DUSP2 mRNA degradation in pancreatic ductal adenocarcinoma[J]. Cell Death \& Disease, 2018, 9(8): 807.

37. Xu R L, He W, Tang J, et al. Primate-specific miRNA-637 inhibited tumorigenesis in human pancreatic ductal adenocarcinoma cells by suppressing Akt1 expression[J]. Experimental Cell Research, 2018, 363(2): 310-314.

38. Abolfazl A, Amir A, Le L T Y, et al. AKT1 and SELP Polymorphisms Predict the Risk of Developing Cachexia in Pancreatic Cancer Patients[J]. PLoS ONE, 2014, 19(9): 108057.

39. Schofield H K, Zeller J, Espinoza C, et al. Mutant p53R270H drives altered metabolism and increased invasion in pancreatic ductal adenocarcinoma[J]. JCI Insight, 2018, 3(2): 97422.

40. Qian Z R, Rubinson D A, Nowak J A, et, al. Association of Alterations in Main Driver Genes With Outcomes of Patients With Resected Pancreatic Ductal Adenocarcinoma[J]. JAMA Oncology, 2018, 4(3): 173420.

41. Takano S, Fukasawa M, Kadokura M, et al. Next-Generation Sequencing Revealed TP53 Mutations to Be Malignant Marker for Intraductal Papillary Mucinous Neoplasms That Could Be Detected Using Pancreatic Juice[J]. Pancreas, 2017, 46(10): 1281- 1287.

42. Maity G, Haque I, Ghosh A, et al. The MAZ transcription factor is a downstream target of the oncoprotein Cyr61/CCN1 and promotes pancreatic cancer cell invasion via CRAF-ERK signaling[J]. Journal of Biological Chemistry, 2018, 293(12): 4334- 4349.

43. Geismann C, Arlt A, Bauer I, et al. Binding of the transcription factor Slug to the L1CAM promoter is essential for transforming TGF- $\beta$-induced L1CAM expression in human pancreatic ductal adenocarcinoma cells[J]. International Journal of Oncology, 2011, 38(1): 257- 266.

44. Liu Y, Zhang W, Wang XJ, et al. Antitumor effect of Kanglaite ${ }^{\circledR}$ injection in human pancreatic cancer xenografts[J]. BMC Complementary \& Alternative Medicine, 2014, 14:228. 
45. Pan $P, W u$ Y, Guo $Z Y$, et al. Antitumor activity and immunomodulatory effects of the intraperitoneal administration of Kanglaite in vivo in Lewis lung carcinoma[J]. Journal of Ethnopharmacology, 2012, 143(2): 680-685.

46. Wang Y, Zhang C, Zhang S, et al. Kanglaite sensitizes colorectal cancer cells to Taxol via NF-KB inhibition and connexin 43 upregulation[J]. Scientific Reports, 2017, 7(1): 1280.

47. Yang $X$, Cheng $Z$, Zhang $L$, et al. Prion Protein Family Contributes to Tumorigenesis via Multiple Pathways[J]. Advances in Experimental Medicine \& Biology, 2017, 1018: 207- 224.

48. Karpiński TM. The Microbiota and Pancreatic Cancer[J]. Gastroenterology Clinics of North America, 2019, 48(3): 447- 464.

49. Chen $Y, B a i X$, Zhang $Q$, et al. The hepatitis $B$ virus $X$ protein promotes pancreatic cancer through modulation of the PI3K/AKT signaling pathway[J]. Cancer Letters, 2016, 380(1): 98- 105.

50. Usami M, Kikuchi S, Takada K, et al. FoxO3a activation by HDAC class lla inhibition induces cell cycle arrest in pancreatic cancer cells[J]. Pancreas, 2020, 49(1): 135- 142.

51. Gasparini G, Pellegatta M, Crippa S, et al. Nerves and Pancreatic Cancer: New Insights into a Dangerous Relationship[J]. Cancers, 2019, 11(7): 893.

52. Zhu Z W, Friess H, Wang L, et al. Nerve Growth Factor Exerts Differential Effects on the Growth of Human Pancreatic Cancer Cells[J]. Clinical Cancer Research, 2001, 7(1): 105- 112.

53. Xin B, He X, Wang J, et al. Nerve growth factor regulates CD133 function to promote tumor cell migration and invasion via activating ERK1/2 signaling in pancreatic cancer[J]. Pancreatology, 2016, 16(6): 1005- 1014.

54. Nomura A, Majumder K, Giri B, et al. Inhibition of NF-kappa B pathway leads to deregulation of epithelial-mesenchymal transition and neural invasion in pancreatic cancer[J]. Laboratory investigation, 2016, 96(12): 1268- 1278.

55. Handra-Luca A, Lesty C, Hammel P, et al. Biological and Prognostic Relevance of Mitogen-Activated Protein Kinases in Pancreatic Adenocarcinoma[J]. Pancreas, 2012, 41(3): 416- 421.

56. Yan Z L, Ohuchida k, Fei S, et al. Inhibition of ERK1/2 in cancer-associated pancreatic stellate cells suppresses cancer-stromal interaction and metastasis[J]. Journal of Experimental \& Clinical Cancer Research, 2019, 38(1): 221.

57. Xu T, Ding J, Ge H, et al. Effects of VCP979 Novel p38 Mitogen Activated Protein Kinase Inhibitor on Progression of Pancreatic Cancer in Mouse Model With Diabetic Conditions[J]. Journal of biomedical nanotechnology, 2019, 15(6): 1325- 1333.

58. Wang S, Lei Y Q, Cai Z L, et al. Girdin regulates the proliferation and apoptosis of pancreatic cancer cells via the PI3K/Akt signalling pathway[J]. Oncology Reports, 2018, 40(2): 599- 608.

59. Zhang YL, Zhang R, Luo GP, et al. Long noncoding RNA SNHG1 promotes cell proliferation through PI3K/AKT signaling pathway in pancreatic ductal adenocarcinoma[J]. Journal of Cancer, 2018, 9(15): 2713- 2722. 
60. Wang L, Wang F, Na L, et al. LncRNA AB209630 inhibits gemcitabine resistance cell proliferation by regulating PI3K/AKT signaling in pancreatic ductal adenocarcinoma[J]. Cancer Biomarkers, 2018, 22(1): 169- 174.

61. Lee JT Jr, McCubrey JA. The Raf/MEK/ERKsignal transduction cascade as a target for chemotherapeutic intervention in leukemia.[J].Leukemia, 2002, 16(4): 486- 507.

62. Aksamitiene E, Kiyatkin A, Kholodenko B N. Cross-talk between mitogenic Ras/MAPK and survival PI3K/Akt pathways: a fine balance[J]. Biochemical Society Transactions, 2012, 40(1): 139- 146.

63. Shankar E, Weis M C, Avva J, et al. Complex Systems Biology Approach in Connecting PI3K-Akt and NF-KB Pathways in Prostate Cancer[J]. Cells, 2019, 8(3): 201.

\section{Figures}

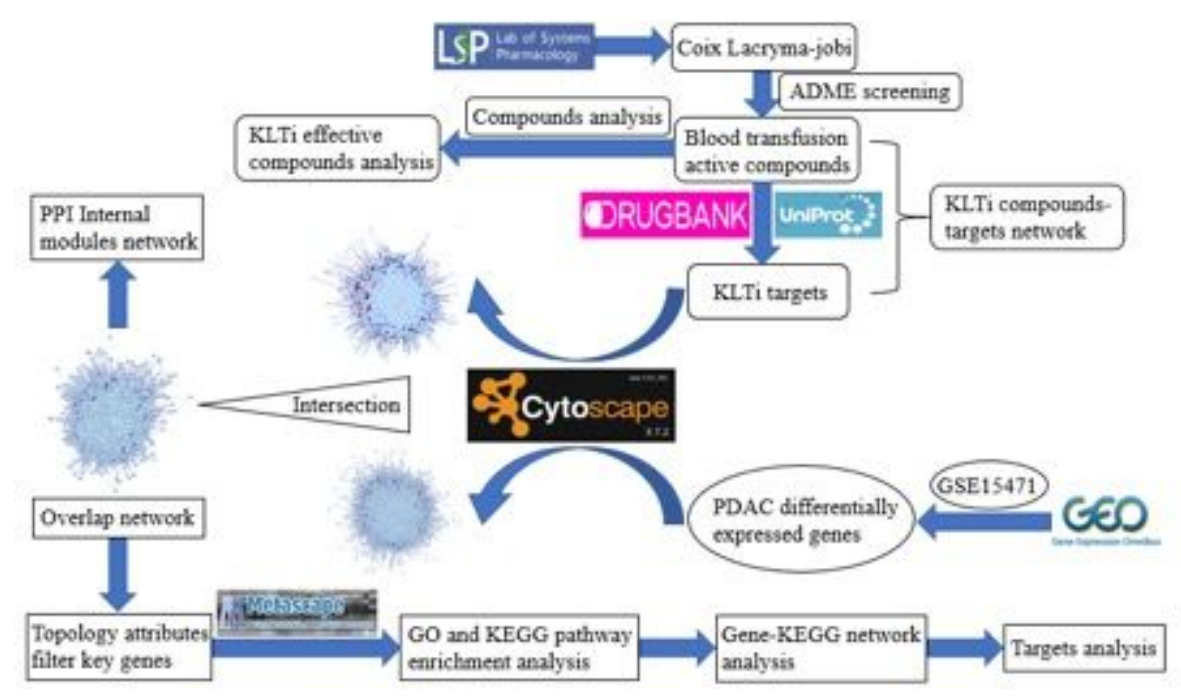

\section{Figure 1}

Workflow of network pharmacology analysis. 


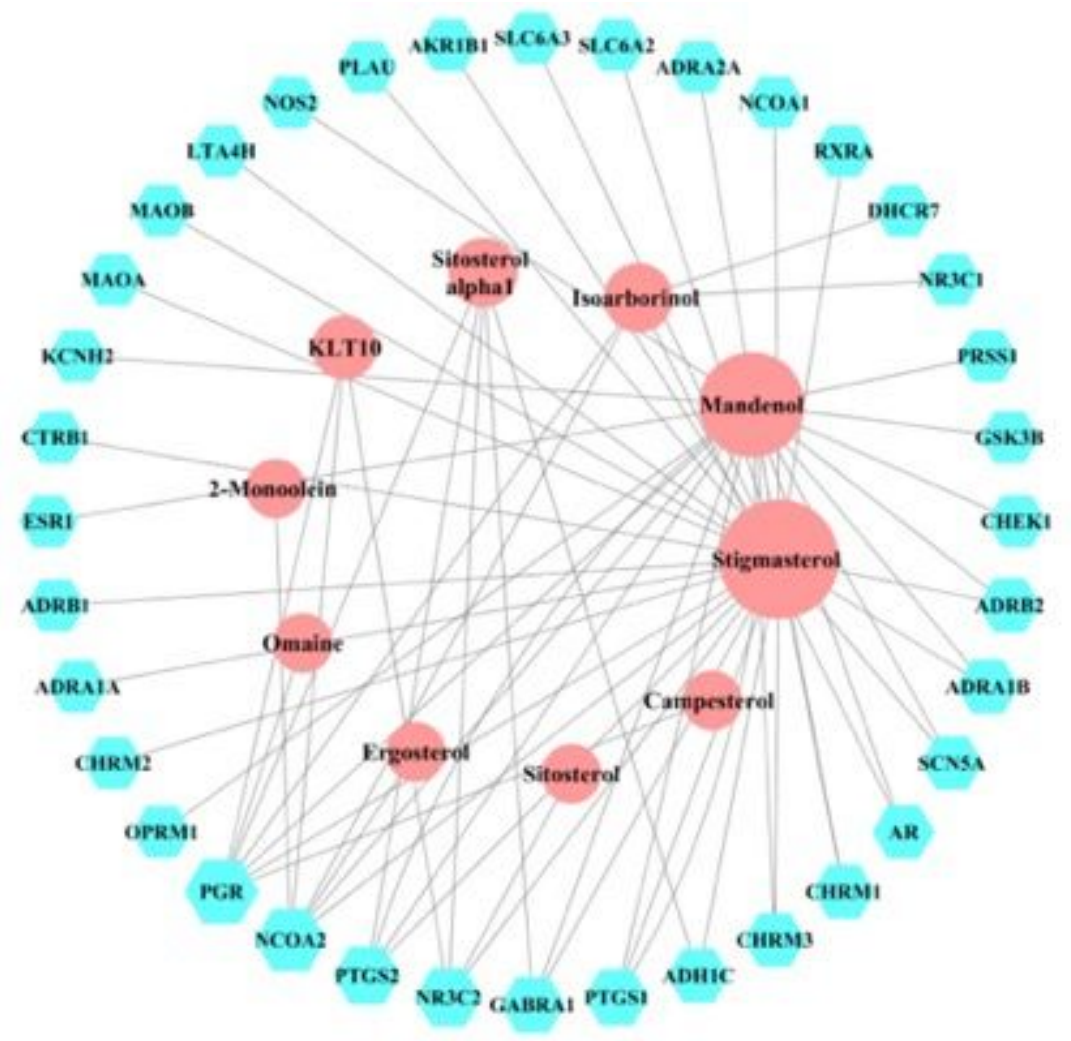

Figure 2

Compound-target network of Kanglaite injection. (Notes: Blue hexagons represent targets; pink circles represent compounds)

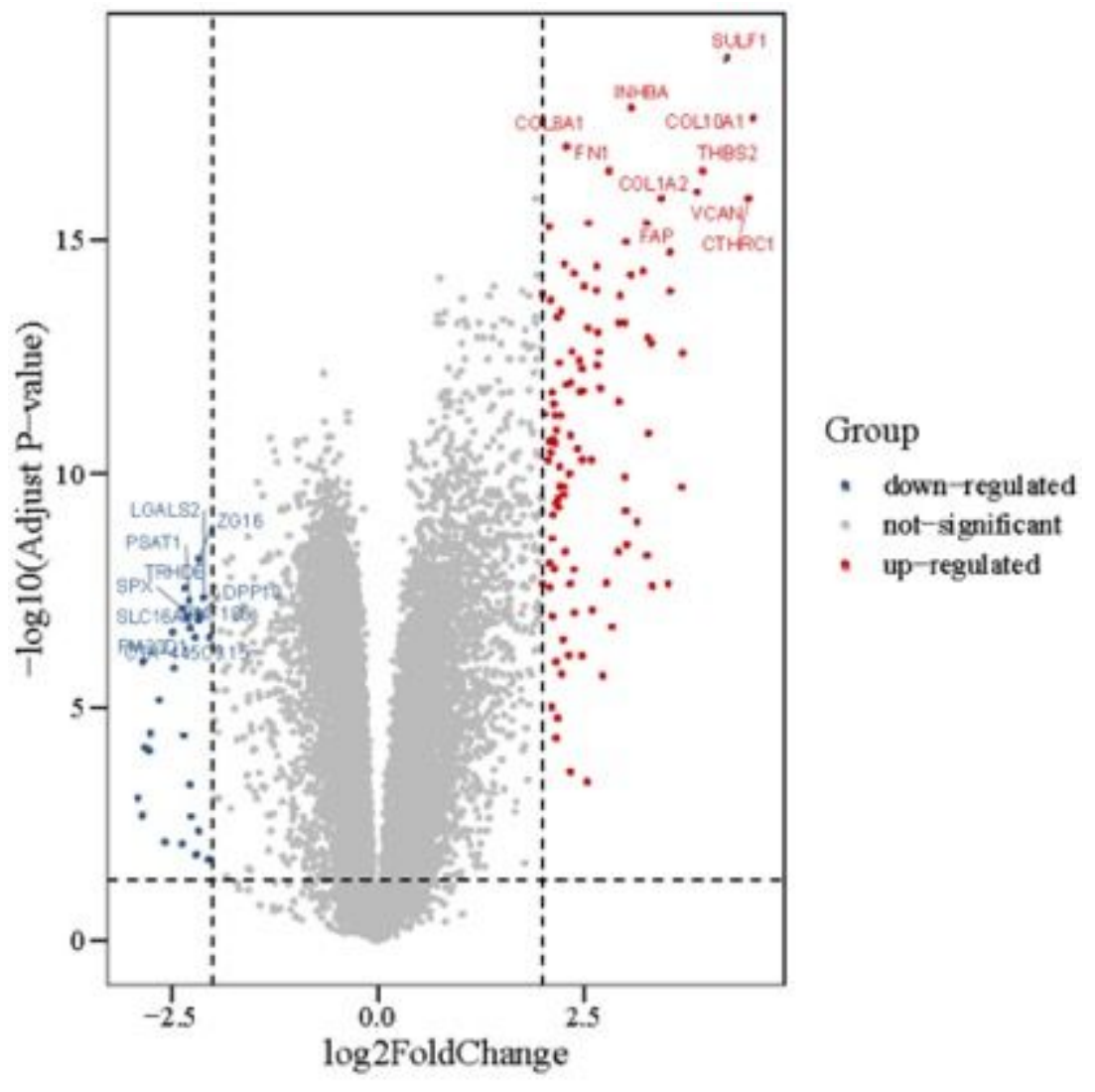




\section{Figure 3}

Volcano plot of differentially expressed genes. (The abscissa represents the fold changes in gene expression and the ordinate represents the statistical significance of the variations in gene expression. The red dots represent significantly differentially expressed genes)

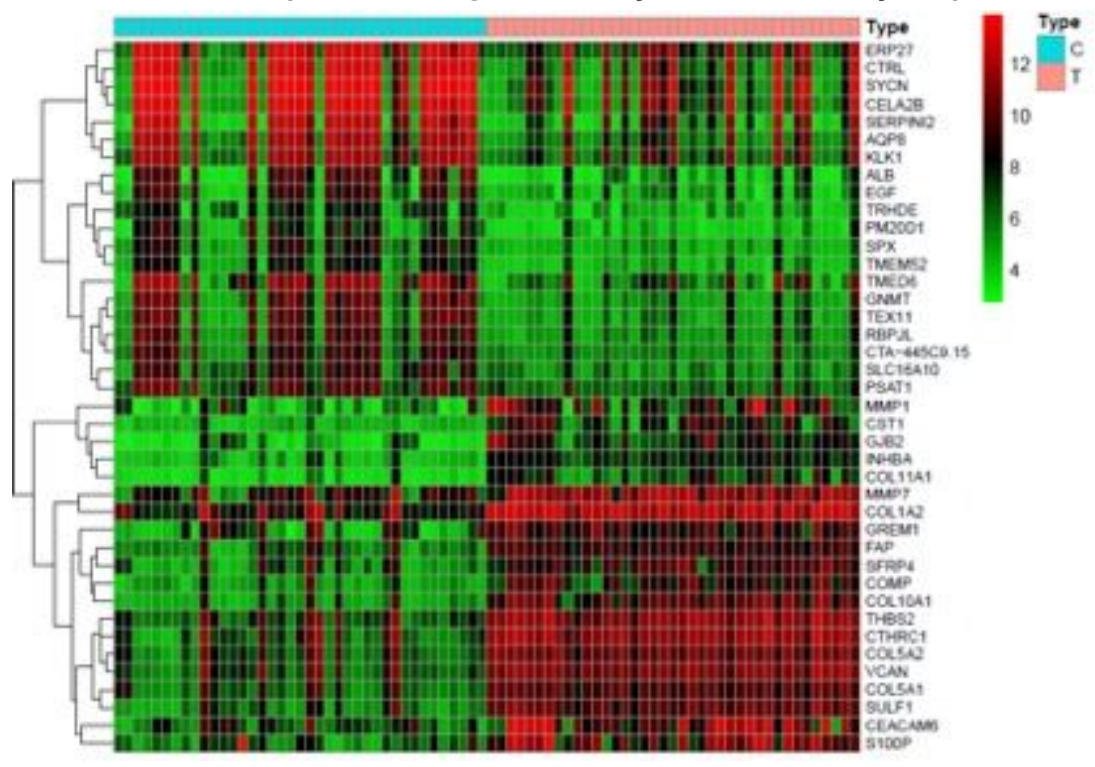

\section{Figure 4}

Heat map of differentially expressed genes.

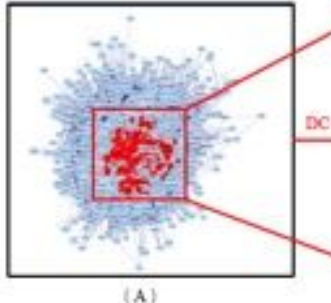

1558 soden mat 40005 edgee

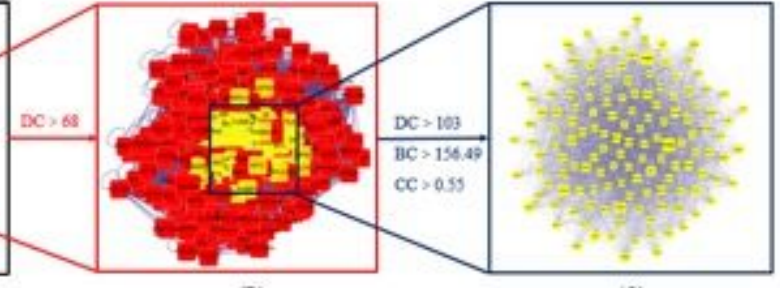

(B)

382 roder and 14158 edpen

193 node and 3593 edper

\section{Figure 5}

The protein-protein interaction (PPI) network of KLTi candidate genes against pancreatic ductal adenocarcinoma (PDAC). ((A) The interactive PPI network of KLTi putative targets and PDAC-related targets. (B) PPI network of significant proteins extracted from A. (C) PPI network of candidate KLTi targets for PDAC therapy extracted from B. DC, degree centrality; BC, betweenness centrality; CC, closeness centrality) 


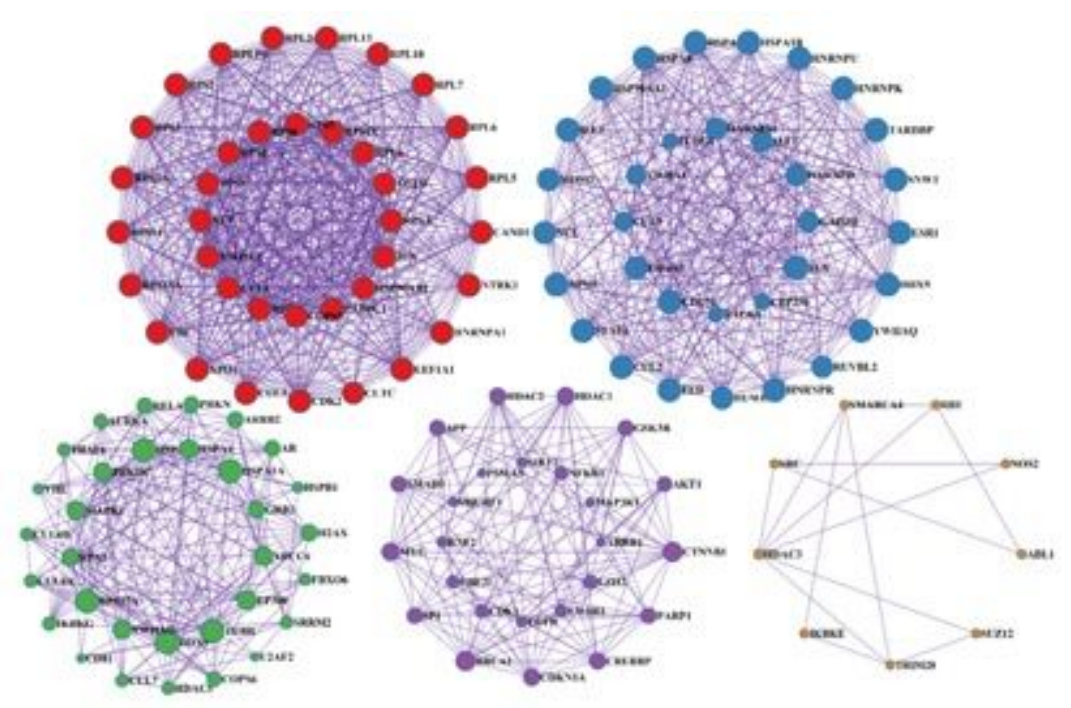

\section{Figure 6}

Kanglaite injection treatment pancreatic ductal adenocarcinoma core protein-protein interaction network internal potential module network. 

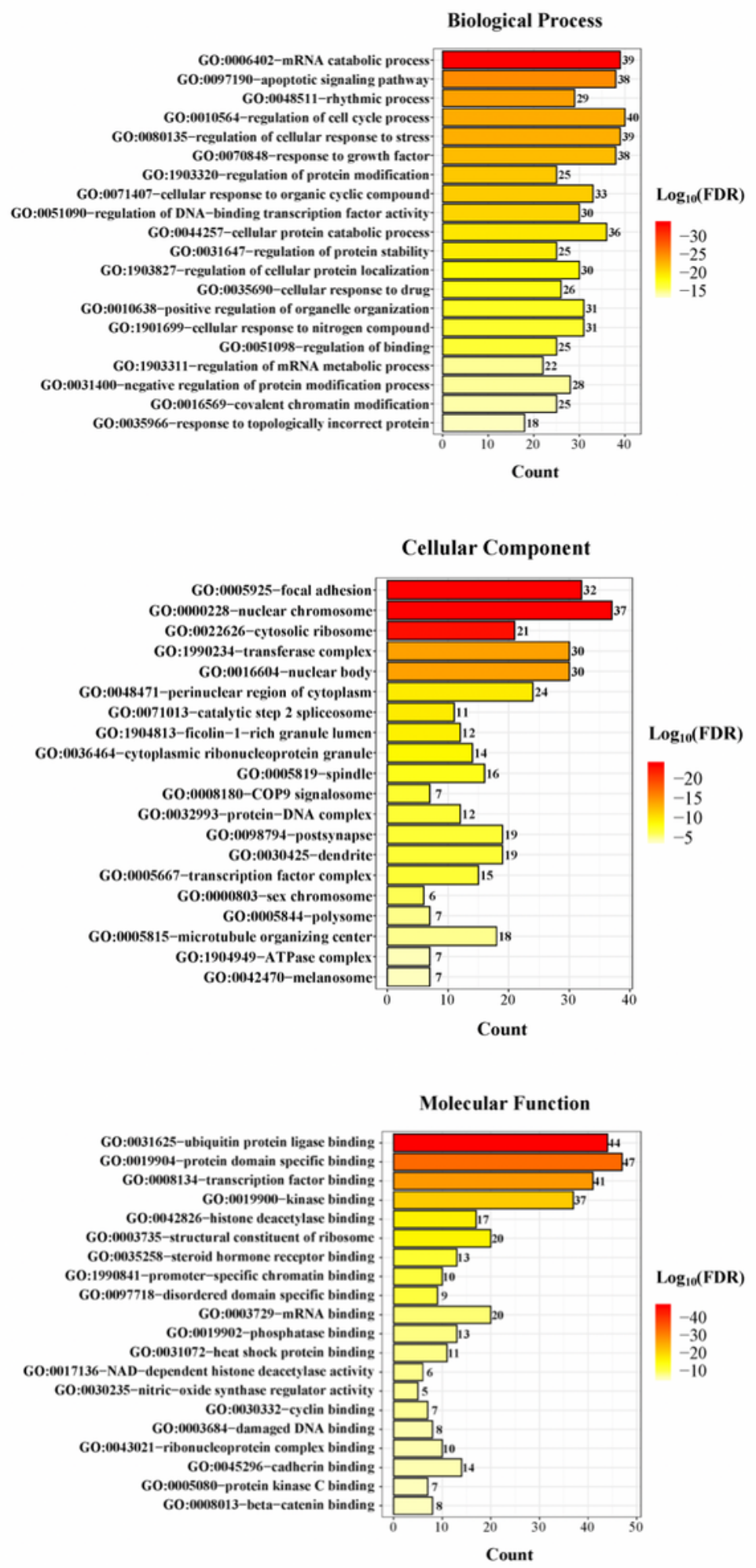

Figure 7

Gene ontology (GO) terms of candidate genes of Kanglaite injection against pancreatic ductal adenocarcinoma. (The left side of the graph is the top GO name, the colors of the bars from orange to red represent the log10(FDR) values (from small to large) and the longer bar represents the gene count of this $\mathrm{GO})$ 


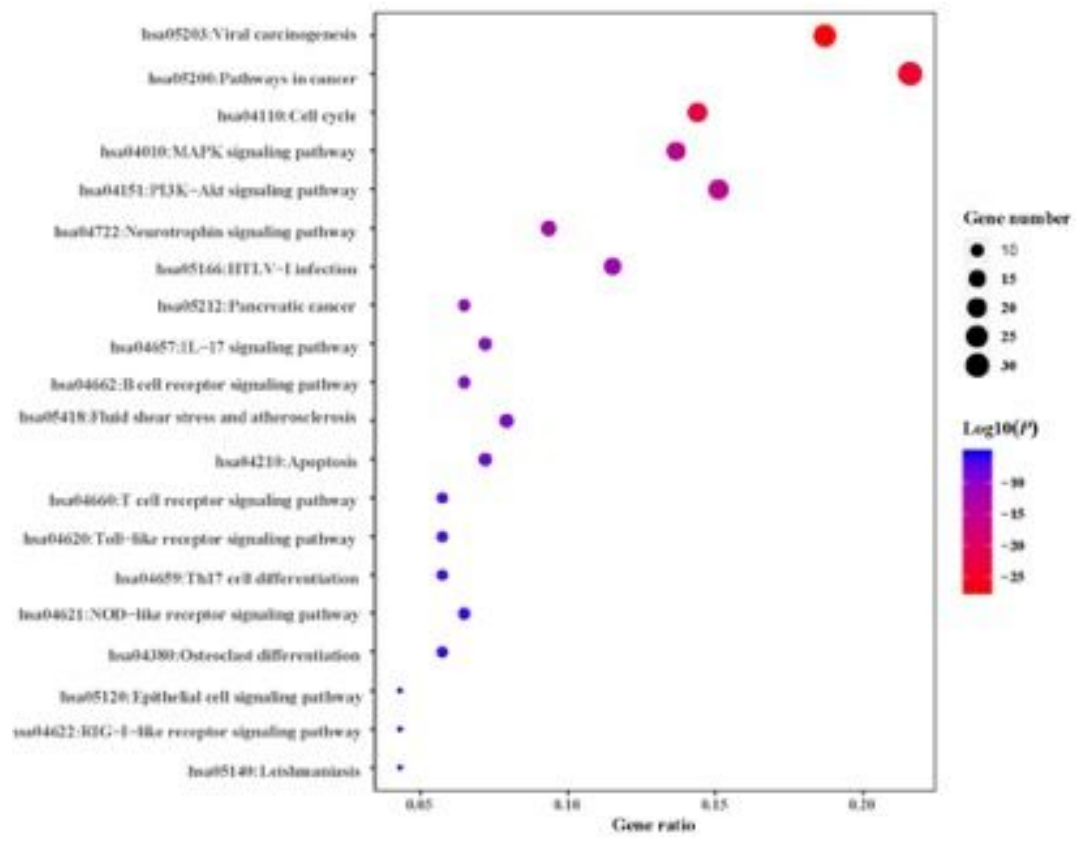

Figure 8

KEGG pathway enrichment of candidate genes upon Kanglaite injection against pancreatic ductal adenocarcinoma. (The size of the bubble represents the number of genes in the pathway, the colors from blue to red represent log10P values (from small to large), the vertical axis represents the top pathway name, and the horizontal axis represents the ratio of the overall input genes in the pathway)

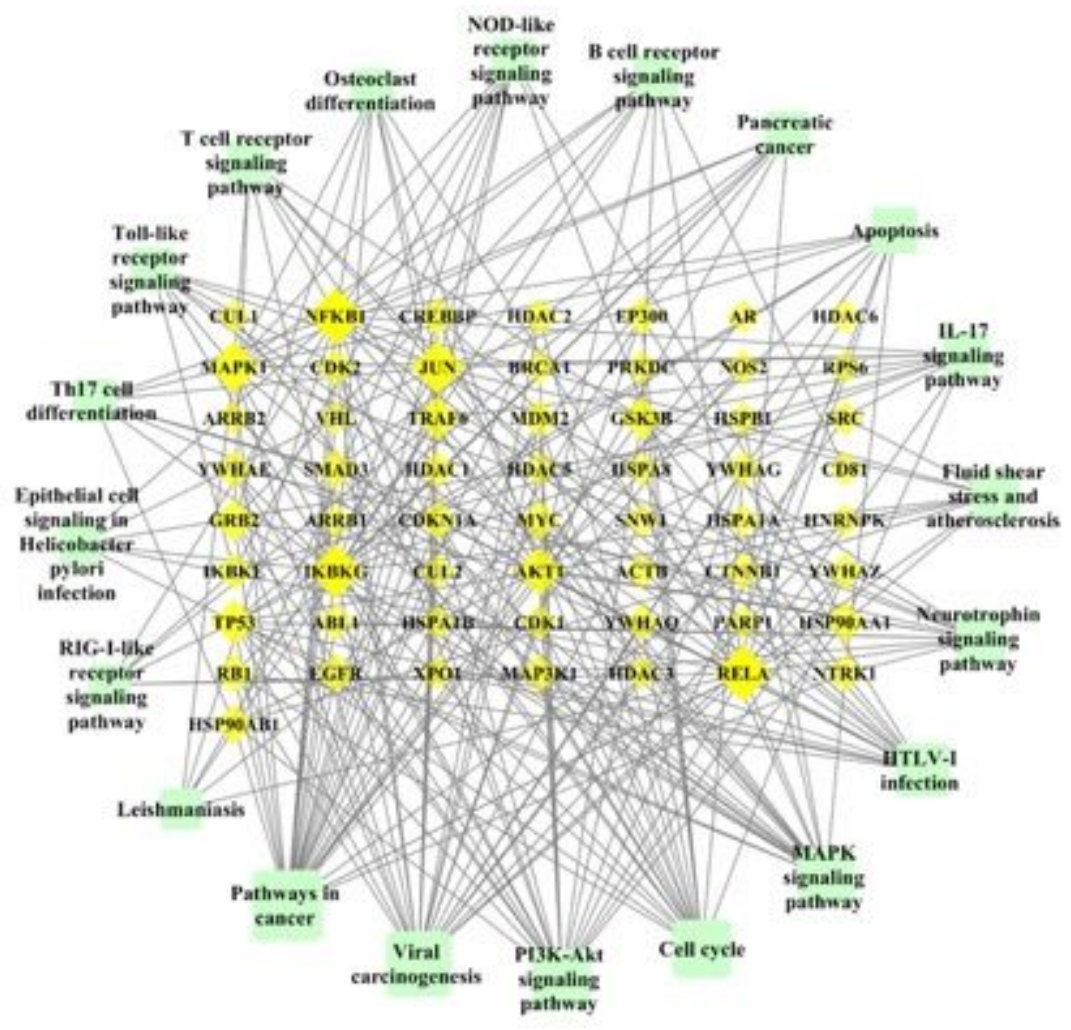

Figure 9 
Gene-Pathway Network of Kanglaite injection against pancreatic ductal adenocarcinoma. (The topological analysis of 20 pathways and 57 genes was carried out with Degree. Yellow diamonds represent target genes, light blue squares represent signaling pathways, and a bigger size represents a larger Degree)

\section{Supplementary Files}

This is a list of supplementary files associated with this preprint. Click to download.

- Table4.ListofGeneontologyGOtermsandKEGGpathways.xIsx

- Table5.NetworkofGenePathwayanalysis.xIsx

- Table1.ListofactivecompoundsandtargetsofKLTi.xlsx

- Table2.ListofdifferentiallyexpressedgenesofPDAC.xIsx

- Table3.ListoftargetsforKLTitreatmentofPDAC.xlsx 